\title{
ARTíCULOS DE INVESTIGACIÓN \\ La carrera de Derecho en Ecuador: Análisis de los planes de estudio en el 2018
}

\author{
A educação jurídica no Equador: Análise dos planos de estudo em 2018 \\ The Law Study in Ecuador: Analyzing the universities' curricula in 2018
}

\author{
Rina Pazos Padilla \\ Universidad Tecnológica Equinoccial, Ecuador
}

Jorge Fabara Espín

Universidade do Minho, Portugal

\begin{abstract}
RESUMEN Se dice que en el Ecuador, así como en varios países de América Latina, las carreras de Derecho tienen un corte positivista, con ausencia de aprendizaje práctico y capacidades críticas, analíticas e interpretativas, así como poca relación con las ciencias sociales y otros campos del conocimiento. No obstante, en el Ecuador la reforma legal de 2010, que instauró un examen en el último año de formación para la evaluación de las carreras, junto a las exigencias del cambio de paradigma constitucional del 2008 en favor de una organización del sistema jurídico, de aplicación directa de derechos constitucionales, debería suponer una nueva dirección que supere el positivismo en su enseñanza superior. Este artículo propone un análisis de los planes de estudio de las carreras de Derecho del Ecuador, según las denominaciones de las asignaturas que los componen, para intentar identificar a qué modelo de educación jurídica responden, así como su núcleo. En términos metodológicos, se seguirá la propuesta del historiador del derecho Robert W. Gordon como marco de referencia para la ubicación de los resultados del análisis del plan de estudios y sobre la cual se generan conclusiones y reflexiones.
\end{abstract}

PALABRAS CLAVE Derecho, educación superior, programas de Derecho, planes de estudio, Ecuador.

RESUMO De longa data tem-se dito que, no Equador e em muitos outros países na América Latina, os currículos de Direito nas universidades são positivistas, carecendo de aprendizado prático e crítico, assim como de capacidade analítica e interpretativa, sem conexão com as outras ciências sociais e os demais campos do conhecimento. Não 
obstante, no Equador, levando-se em conta as mudanças na lei em 2010 que criaram um exame nacional de avaliação de desempenho, como também pelas mudanças constitucionais de 2008 no que diz respeito à aplicação de direitos humanos em todos os níveis, tudo isso pode ter levado a uma nova compreensão capaz de ajudar na superação do positivismo. Este artigo analisa os currículos de direito das universidades equatorianas, de acordo com as preferências dos sujeitos, com o objetivo de identificar com qual paradigma educacional eles se identificam, e também o núcleo da grade curricular. Para cumprir este objetivo, esta abordagem aplicará o modelo dado pelo historiador do direito Robert W. Gordon como referência para classificar os resultados e para auxiliar na chegada de conclusões e reflexões.

PALAVRAS-CHAVE Direito, Educação superior, programas de direito, planos de estudo, Equador.

ABSTRACT It has been said for long time, that in Ecuador as in many other countries in Latin America, the law study programs at universities are positivists, lacking practical learning as well as critical, analytical and interpretative skills or further connection with social sciences and other fields of knowledge. Nevertheless, In Ecuador considering the legal changes enacted in 2010 that allowed for the first time to have a national examination for the law study programs, and also because of the constitutional changes of 2008 regarding the direct application of human rights at all the levels, it should have led to a new direction that might help to overcome positivism. This article makes an analysis of the law curricula from the universities in Ecuador, according to the designations of the subjects, in order to identify to which educational paradigm they belong, as also the program's core. To do so, this approach will apply the model given by the law historian Robert W. Gordon as a reference framework to sort the results and to draw some conclusions and reflections.

KEYWORDS Law, higher education, law study, positivism, curricula, Ecuador, Latin America.

\section{Introducción}

Se dice que en el Ecuador, así como en varios países de América Latina, las carreras de Derecho tienen un corte positivista (Estarellas Velásquez y Velázquez Ávila, 2018), tanto los planes de estudio (Herrero y Perelló Roselló, 2017; Herrera, González y Paniagua, 2018) como las metodologías de enseñanza se enfocan en el aprendizaje de los cuerpos legales y miran a la disciplina como una técnica o herramienta (Elgueta Rosas y Palma González, 2014; Lazo González, 2011; Lobo, 2017; Solari, 2015). Esta situación es heredera de la universidad napoleónica que se implantó con los procesos de independencia de las colonias europeas en América Latina (Aguirre, 1973: 42-43). En otras palabras, se trata de doscientos años de formación jurídica centrada en la ley, y complementariamente en la doctrina, en ausencia del aprendizaje práctico y 
de capacidades críticas (Mourão y otros, 2007; Osorio, 2013; Veres, 2002), analíticas e interpretativas, así como poca relación con las ciencias sociales y otros campos del conocimiento.

Con la tercera ola de reformas a la educación superior en el Ecuador, la de estos últimos años (Díaz Ocampo, 2018), se estableció por primera vez la exigencia de un examen de fin de carrera con el propósito de evaluar los programas de estudio. Este proceso es una atribución del Consejo de Evaluación, Acreditación y Aseguramiento de la Calidad de la Educación Superior (CEAACES), con el que, como parte de la evaluación de la carrera de Derecho, además se prueba los conocimientos mínimos que un estudiante de último año de estudios debe tener. Este examen ha determinado los contenidos que deben asegurarse en los planes de estudio (CEAACES, 2017: 5-6). Así, para atender estas nuevas exigencias, las carreras de Derecho del Ecuador han sido rediseñadas en los últimos años.

Por otro lado, la Constitución del Ecuador del 2008 reafirmó un modelo de Estado constitucional e impulsó una organización del sistema jurídico en el que los derechos constitucionales son de aplicación directa. Esta circunstancia exige que la formación jurídica tome una nueva dirección (González Ibáñez, 2012; González Galván, 2013; Pinilla Rodríguez, Reinoso Vásquez y López Merino, 2017) en la que las capacidades críticas e interpretativas asumen un mayor rol (Giné, 2009), así como el redimensionamiento del papel del juez, quien, además del dominio de las leyes, requiere de la capacidad de razonar e interpretar y valorar los hechos en relación con las normas. Esta nueva visión exige una formación jurídica que supere el positivismo. Luego de los cambios constitucionales y de las políticas de educación superior en la segunda década del siglo XXI, en el Ecuador surge la pregunta: ¿Cuál es la formación jurídica actual en el país? (De Mello Massimino y Pamplona, 2015).

Para intentar contestar esta pregunta, este artículo propone un análisis de los planes de estudio de las carreras de Derecho del Ecuador, según las denominaciones de las asignaturas que los componen, para intentar identificar a qué modelo de educación jurídica responden. Para ello se ha realizado un levantamiento de información en los sitios web de cada universidad, para luego sistematizarla y agruparla según su similitud en 18 grupos y, a partir de esta clasificación, identificar cuál es el núcleo de los planes de estudios y su relación con el examen de evaluación de carrera del CEAACES. También este artículo se propone observar cuáles son los otros grupos de asignaturas predominantes, y aquellas que son escasas, lo que nos permite llegar a conclusiones importantes sobre el tipo de educación jurídica (González Galván, 2013; Betancourt y Prieto, 2017) que imparten las universidades del país.

El artículo está dividido en cuatro partes. En la primera se aborda la clasificación de los modelos de educación jurídica propuesta por el historiador del derecho Robert W. Gordon (2002), lo que sirve como marco de referencia para la ubicación de los resultados del análisis del plan de estudios; en la segunda, se examina brevemente 
cuáles son las principales influencias de los contenidos de la educación jurídica. En la tercera, a través de la metodología antes descrita, se profundiza en la identificación del modelo de educación jurídica en el Ecuador y, en la cuarta parte, se presentan algunas conclusiones y reflexiones.

\section{Modelos de educación jurídica}

Robert W. Gordon (2002), reconocido historiador del derecho de la Universidad de Stanford, plantea que existen diez modelos de educación jurídica y que estos se aplican según el tipo de abogado o abogada que se busca formar, y que van desde profesionales observadores, pasando por aquellos de carácter reformador, activistas públicos, hasta intelectuales.

\section{Modelos tradicionales}

Los modelos tradicionales buscan educar estudiantes para que sean abogados y abogadas observadores. Según la clasificación que realiza Gordon (2002) son cuatro modelos.

El primero modelo es la capacitación de aprendices practicantes, considerada por el autor como la más básica y enfocada en la enseñanza-aprendizaje de procedimientos básicos que se utilizan en escenarios prácticos, así como documentos tipo formularios adaptables a las demandas de los clientes. Este tipo de enseñanza-aprendizaje era común en la capacitación del aprendiz en los estudios jurídicos y fue adoptada por las primeras escuelas de los Estados Unidos como parte central de los planes de estudio.

El segundo modelo tradicional es la enseñanza del derecho positivo, cuyo enfoque apunta específicamente a las reglas escritas en las leyes y códigos en la tradición del civil law y a las doctrinas y reglas de sentencias en el common law. Este tipo de formación crea un profesional pasivo que acude a la norma escrita para informar lo que ahí se señala a sus clientes; no adquiere capacidades creativas o interpretativas del derecho que lo constituyan un agente activo de creación jurídica.

El tercer modelo tradicional es el que enseña a pensar como abogados. En este modelo se forma a los estudiantes con varios de los métodos de la profesión, como la argumentación o la retórica. Se ubica frecuentemente en las clínicas jurídicas desarrolladas especialmente en Estados Unidos a través del método de casos. La intención es que los estudiantes aprendan las lógicas habituales de los profesionales del derecho, así como habilidades de razonamiento pragmático para la resolución de problemas.

El cuarto modelo tradicional es el de la enseñanza de la ciencia legal, esto es, la educación basada en que el derecho es un sistema cerrado de principios y reglas coherente: se desarrolla la habilidad de razonar deductiva e inductivamente entre 
principios y casos particulares. Este modelo se observa con mayor predominio en los países con tradición de civil law y sobre todo en el siglo XIX.

\section{El derecho como política}

Los modelos que miran al derecho como política son tres y buscan formar abogadas y abogados reformadores, según el propio Gordon (2002). En el primero de estos modelos se aborda la enseñanza de la política como suplemento a la educación tradicional, perspectiva impulsada por el movimiento realista del derecho que influyó desde la mitad del siglo XX en las escuelas de derecho de los Estados Unidos.

El segundo modelo aborda la política como una técnica de ingeniería legal-tecnocrática en la que la política se enseña para que apoye a las tareas legales establecidas, así como lo serían las disciplinas contables, económicas, estadísticas, psiquiátricas o psicológicas para las correspondientes especializaciones de los abogados.

El tercero se enfoca en enseñar la disciplina como el arte de gobernar para los juristas, en el que la política toma el lugar del fundamento del derecho y este se transforma en una herramienta para su ejecución. Esta visión fue planteada en el siglo XVIII por pensadores como Adam Smith y David Hume. Se trata de ver al derecho como un mecanismo de mejoras que dejarán atrás regímenes obsoletos, luego que estos se adapten a los valores éticos de una sociedad comercial moderna.

\section{Otras perspectivas}

También se proponen otras perspectivas que procuran contar con profesionales activistas o intelectuales. En estas, el derecho se aborda como objeto de estudio desde otros prismas, como desde el punto de vista filosófico, histórico, sociológico, cultural, entre otros. Los estudios críticos y la educación del derecho para el cambio social han tenido escasa aplicación, pero existen.

\section{La influencia de los contenidos de las carreras de Derecho}

En cuanto a los contenidos, estos dan pistas del modelo e influencia ideológica que subyace en la estructura curricular. La literatura señala tres grandes bloques de asignaturas de la formación jurídica.

En el primero, las asignaturas de Derecho Civil, Derecho Penal y Derecho Procesal se asientan sobre la ideología del laissez faire del capitalismo del siglo XIX. Esto se observa en el Ecuador después de la Independencia de la corona española con la intensificación de las cátedras de Derecho Civil que restaron espacio a la enseñanza del Derecho Canónico propio de la colonia (Aguirre, 1973: 44).

En el segundo, las asignaturas sobre Derecho Administrativo y Teoría del Estado, Derecho Tributario, entre otras, tienen como base el programa del New Deal en el 
que se transmite que es razonable una intervención estatal en el mercado (Kennedy, 2004: 127).

En el tercero, las asignaturas de Derecho Social responden a los resultados de los movimientos de los años sesenta del siglo XX (Pérez Lledó, 2001: 92). En este período, en los Estados Unidos también cobra fuerza el movimiento de enseñanza jurídica a través de clínicas, que llega a América Latina en los años setenta, pero solo en los noventa tiene impacto en la estructura curricular de las carreras en la región (Witker, 2007: 184-187), con un énfasis en la justicia social que buscan una formación práctica basada en casos reales (Londoño Toro, 2016: 123).

Las asignaturas relacionadas con las humanidades y las ciencias sociales no aparecen en estos bloques, sin embargo, sí suelen formar parte del currículum, aunque en un lugar secundario, muchas veces como materias optativas (Pérez Lledó, 2001: 91).

\section{El modelo de educación jurídica en el Ecuador}

Desde la instalación de las escuelas de derecho, el modelo predominante en América Latina fue el de corte tradicional de enseñanza del derecho positivo (Pérez Perdomo, 2007: 260), es decir, se forma abogados y abogadas con un rol de espectadores, en el que la tarea intelectual es saber las normas, lo cual desde la perspectiva de Kennedy (2004: 117) aporta a que se considere como «natural, eficiente y justo» que tanto los estudios, la profesión jurídica y la sociedad en la que se desenvuelven mantengan una organización jerárquica y de dominación.

$\mathrm{Al}$ no identificarse estudios específicos para el Ecuador, podría considerarse que estas afirmaciones abarcan a la educación jurídica ecuatoriana; sin embargo, es propósito de este trabajo intentar confirmar esta hipótesis a través del análisis de los planes de estudios vigentes en las universidades del país.

\section{El examen de evaluación de carrera del CEAACES}

Por disposición de la Ley Orgánica de Educación Superior del año 2010, los estudiantes ecuatorianos deben rendir un examen en el último año de la carrera con el fin de evaluar su formación. El examen se centra en los conocimientos adquiridos durante la carrera. Para el caso de la carrera de Derecho se establecieron siete componentes: 1) Derecho Constitucional; 2) Derecho Procesal; 3) Derecho Penal; 4) Derecho Civil; 5) Derecho Administrativo; 6) Introducción al Derecho y Filosofía del Derecho; y 7) Derecho Laboral (CEAACES, 2017: 5-6).

Las preguntas del examen son de opción múltiple o del tipo en el que se pide completar palabras. A pesar de que no existe una descripción extendida sobre el diseño del examen, en la guía emitida por el CEAACES (2017: 5-6) se señala que es un examen de conocimiento, y de los ejemplos de preguntas que ahí se indican se pue- 
de colegir que se centran en el conocimiento sobre principios y normas. No evalúa la capacidad crítica sobre la disciplina ni las habilidades prácticas e investigativas. En términos de la taxonomía de Bloom (1956: 62-68), el examen mide la primera clasificación de la dimensión cognitiva del aprendizaje, que es conocer, sin que se haya podido constatar que también mida comprensión, aplicación, análisis, creación o síntesis y evaluación. A continuación, transcribimos un ejemplo de las preguntas que se plantean:

El delito de latrocinio supone la muerte con ocasión del robo o, dicho de otra forma, robo con muerte. La estructura típica se encuentra en el artículo 189 del COIP [Código Orgánico Integral Penal], en el sexto inciso, la norma reza: «La persona que mediante amenazas o violencias sustraiga o se apodere de cosa mueble ajena, sea que la violencia tenga lugar antes del acto para facilitarlo, en el momento de cometerlo o después de cometido para procurar impunidad, será sancionada con pena privativa de libertad de cinco a siete años [...] Si a consecuencia del robo se ocasiona la muerte, la pena privativa de libertad será de veintidós a veintiséis años».

La posibilidad que en un mismo tipo penal se verifique una conducta que lesiona simultánea o sucesivamente dos o más bienes jurídicos, se conoce como:

a) Principio de legalidad

b) Concurso ideal de infracciones

c) Concurso real de infracciones

d) Principio de consunción (CEAACES, 2017: 11).

Estos contenidos mínimos y el tipo de preguntas del examen señalado establecen la orientación de la formación jurídica en el Ecuador, y ratifican los tres bloques de contenidos que se mencionan en la sección anterior, pero dejando de lado los componentes prácticos, con lo cual se podría afirmar que, desde la óptica de la calidad, la formación es de corte positivista según la clasificación de Gordon (2002).

Aunque queda claro cuál es la concepción del núcleo duro de la formación jurídica de un abogado o una abogada para el ente rector de la calidad de la educación superior en el Ecuador, además de brindar la educación para cumplir con estos requerimientos, las universidades en ejercicio de la autonomía que poseen pueden incorporar contenidos adicionales y metodologías que maticen o varíen la orientación antes mencionada. Para identificar cuáles son las características y patrones de los contenidos curriculares, en la siguiente sección se realizará un análisis de los planes de estudios de todas las universidades ecuatorianas que ofertan la carrera de Derecho. De esta manera, se podrá observar si los requerimientos de contenidos del CEAACES condicionan decisoriamente la orientación de la formación jurídica o si se ha aprovechado de la posibilidad de incorporar nuevas perspectivas en cuanto al perfil de los futuros abogados y abogadas. 


\section{Caracterización de las universidades que ofertan Derecho en el Ecuador}

En el Ecuador existen 29 universidades con oferta académica vigente en Derecho en el nivel de pregrado. De estas, 19 son de naturaleza jurídica privada, es decir, el 66\% del total (tabla 1$).{ }^{1}$

La cobertura geográfica de las carreras de Derecho es mayoritaria en la serranía del país, con 28 instituciones que ofertan la carrera. En la costa hay una presencia de 15 instituciones y en la Amazonía se cuenta con una universidad. La región insular no tiene esta oferta. En la sierra, la principal provincia que cuenta con presencia institucional es Pichincha, en la ciudad de Quito, con 9 universidades de 28: esto se explica por ser esta la capital del Estado y, por tanto, concentrar a los máximos órganos y funciones estatales, además de tener una alta población: es el segundo cantón más poblado del país (INEC, 2017). La mayoría de las universidades que ofertan Derecho en esta región son privadas.

El fenómeno de concentración de las instituciones en las grandes ciudades es mayor en la región Costa. En la provincia del Guayas se concentran 7 de 15 universidades con oferta académica en Derecho de toda esa región. Guayaquil es la ciudad más grande del país con mayor población y dinamismo económico (INEC, 2018). Otra característica es que 11 de estas instituciones son de naturaleza privada, no obstante, aunque pareciera que existe un predominio de la educación universitaria privada en este campo de conocimiento, la Universidad de Guayaquil, con la mayor cantidad de alumnos del Ecuador, es pública.

Mientras tanto en la Amazonía, por ser su población menor en relación con las otras dos regiones (INEC, 2018), tiene apenas una universidad que oferta Derecho: la Universidad Regional Autónoma de los Andes.

\section{Composición de los planes de estudio de las carreras de Derecho del Ecuador}

En virtud de la autonomía universitaria consagrada en la Constitución de la República, las universidades ecuatorianas pueden diseñar sus carreras de manera independiente, no obstante, los proyectos nuevos tanto como los rediseños deben ser aprobados por el Consejo de Educación Superior, con base a criterios de pertinencia y calidad.

Para el análisis de los planes de estudio de las 28 universidades que ofertan Derecho en el país y a cuya información ha sido posible acceder, ${ }^{2}$ se ha clasificado a las asignaturas en 18 grupos, considerando tres criterios: 1) la clasificación de campos

1. Los datos de esta sección están tomados del sitio de la Secretaría de Educación Superior, Ciencia, Tecnología e Innovación (SENESCYT) de Ecuador, disponibles en www.educacionsuperior.gob.ec.

2. El plan de estudios de la Universidad del Pacífico no se encuentra disponible en su página web. 
Tabla 1. Universidades con oferta académica de pregrado en Derecho

\begin{tabular}{|c|c|c|}
\hline Número & Nombre & Naturaleza \\
\hline 1 & Pontifica Universidad Católica del Ecuador & Privada \\
\hline 2 & Universidad Católica de Cuenca & Privada \\
\hline 3 & Universidad Católica Santiago de Guayaquil & Privada \\
\hline 4 & Universidad de Especialidades Espíritu Santo & Privada \\
\hline 5 & Universidad de las Américas & Privada \\
\hline 6 & Universidad de los Hemisferios & Privada \\
\hline 7 & Universidad de Otavalo & Privada \\
\hline 8 & Universidad del Azuay & Privada \\
\hline 9 & Universidad del Pacífico & Privada \\
\hline 10 & Universidad Internacional del Ecuador & Privada \\
\hline 11 & Universidad Laica Vicente Rocafuerte & Privada \\
\hline 12 & Universidad Metropolitana & Privada \\
\hline 13 & Universidad Regional Autónoma de los Andes & Privada \\
\hline 14 & Universidad San Francisco de Quito & Privada \\
\hline 15 & Universidad San Gregorio & Privada \\
\hline 16 & Universidad SEK & Privada \\
\hline 17 & Universidad Técnica Particular de Loja & Privada \\
\hline 18 & Universidad Tecnológica Ecotec & Privada \\
\hline 19 & Universidad Tecnológica Indoamérica & Privada \\
\hline 20 & Universidad Central del Ecuador & Pública \\
\hline 21 & Universidad de Cuenca & Pública \\
\hline 22 & Universidad de Guayaquil & Pública \\
\hline 23 & Universidad Estatal del Bolívar & Pública \\
\hline 24 & Universidad Estatal Península de Santa Elena & Pública \\
\hline 25 & Universidad Laica Eloy Alfaro & Pública \\
\hline 26 & Universidad Nacional de Chimborazo & Pública \\
\hline 27 & Universidad Nacional de Loja & Pública \\
\hline 28 & Universidad Técnica de Ambato & Pública \\
\hline 29 & Universidad Técnica de Machala & Pública \\
\hline
\end{tabular}

Elaboración propia con datos tomados de SENESCYT.

amplios y específicos de la Clasificación Internacional Normalizada de la Educación (CINE) 2013 (Unesco, 2013: 17); 2) las principales ramas del Derecho (Suárez, 2004: 119); y 3) las técnicas auxiliares para el ejercicio del Derecho.

- Grupo 1: Educación Comercial y Administración, envuelve asignaturas como Contabilidad, Gestión y Administración que se encuentran dentro del campo detallado correspondiente al campo específico o41 de la CINE (Unesco, 2013). 
Tabla 2. Universidades con oferta académica en pregrado en Derecho en la región Sierra

\begin{tabular}{|lll|}
\hline Provincia & Cantón & Universidad \\
\hline Azuay & Cuenca & Universidad del Azuay \\
\hline Azuay & Cuenca & Universidad Católica de Cuenca \\
\hline Azuay & Cuenca & Universidad de Cuenca \\
\hline Bolivar & Guaranda & Universidad Estatal del Bolívar \\
\hline Cañar & Azogues & Universidad Católica de Cuenca \\
\hline Cañar & La Troncal & Universidad Católica de Cuenca \\
\hline Carchi & Tulcán & Universidad Regional Autónoma de los Andes \\
\hline Chimborazo & Riobamba & Universidad Nacional de Chimborazo \\
\hline Chimborazo & Riobamba & Universidad Regional Autónoma de los Andes \\
\hline Imbabura & Ibarra & Pontifica Universidad Católica del Ecuador \\
\hline Imbabura & Ibarra & Universidad Regional Autónoma de los Andes \\
\hline Imbabura & Otavalo & Universidad de Otavalo \\
\hline Loja & Loja & Universidad Técnica Particular de Loja \\
\hline Loja & Loja & Universidad Nacional de Loja \\
\hline Loja & Loja & Universidad Internacional del Ecuador \\
\hline Pichincha & Quito & Universidad SEK \\
\hline Pichincha & Quito & Universidad de las Américas \\
\hline Pichincha & Quito & Universidad San Francisco de Quito \\
\hline Pichincha & Quito & Pontifica Universidad Católica del Ecuador \\
\hline Pichincha & Quito & Universidad de los Hemisferios \\
\hline Pichincha & Quito & Universidad Metropolitana \\
\hline Pichincha & Quito & Universidad Internacional del Ecuador \\
\hline Pichincha & Quito & Universidad Central del Ecuador \\
\hline Pichincha & Quito & Universidad Tecnológica Indoamérica \\
\hline Tungurahua & Ambato & Universidad Regional Autónoma de los Andes \\
\hline Tungurahua & Ambato & Universidad Tecnológica Indoamérica \\
\hline Tungurahua & Pontifica Universidad Católica del Ecuador \\
\hline Tungurahua & Universidad Técnica de Ambato \\
\hline
\end{tabular}

Elaboración propia con datos tomados de SENESCYT.

- Grupo 2: Artes, se refiere a asignaturas correspondientes al campo específico o21 de la CINE (Unesco, 2017).

- Grupo 3: Ciencia Jurídica, considera asignaturas como Teoría del Derecho, Teoría de la Norma, Derecho Comparado (Núñez Vaquero, 2019: 610-614).

- Grupo 4: Comunicación y Lenguaje abarca asignaturas como Comunicación Oral y Escrita, Lectura Crítica, Redacción Jurídica o Idiomas. 
Tabla 3. Universidades con oferta académica en pregrado en Derecho en la región Costa

\begin{tabular}{|lll|}
\hline Provincia & Cantón & Universidad \\
\hline El Oro & Machala & Universidad Técnica de Machala \\
El Oro & Machala & Universidad Metropolitana \\
Guayas & Guayaquil & Universidad Laica Vicente Rocafuerte \\
Guayas & Guayaquil & Universidad del Pacífico \\
Guayas & Guayaquil & Universidad Católica Santiago de Guayaquil \\
Guayas & Guayaquil & Universidad Metropolitana \\
Guayas & Guayaquil & Universidad de Guayaquil \\
Guayas & Samborondón & Universidad Tecnológica Ecotec \\
Guayas & Samborondón & Universidad de Especialidades Espíritu Santo \\
Los Ríos & Babahoyo & Universidad Regional Autónoma de los Andes \\
Los Ríos & Quevedo & Universidad Regional Autónoma de los Andes \\
Manabí & Portoviejo & Universidad San Gregorio \\
Manabí & Manta & Universidad Laica Eloy Alfaro \\
Santa Elena & La Libertad & Universidad Estatal Península de Santa Elena \\
Santo Domingo & Santo Domingo & Universidad Regional Autónoma de los Andes \\
Elaboración propia con datos tomados de SENEScYT. & \\
\hline
\end{tabular}

- Grupo 5: Derecho privado abarca las asignaturas de Derecho Sustantivo Privado, como Derecho Civil, Societario y Mercantil (García Arellano, 2004: 16).

- Grupo 6: Derecho Procesal envuelve las asignaturas procesales, como Derecho Procesal Civil, Penal o Laboral (García Arellano, 2004: 16).

- Grupo 7: Derecho Público incluye a las asignaturas de Derecho Sustantivo en el ámbito público, como Derecho Constitucional, Penal, Administrativo (García Arellano, 2004: 16).

- Grupo 8: Derecho Social tiene relación con asignaturas como Derecho de Cooperativas, Derecho Laboral, de Menores (García Arellano, 2004: 16).

- Grupo 9: Derechos Humanos, incluye asignaturas con esa misma denominación u otras tales como Derechos Culturales que son parte de los Derechos Humanos (Donnelly, 2013: 28-32).

- Grupo 10: Filosofía contiene asignaturas como Filosofía del Derecho, Deontología Jurídica, Lógica Jurídica, Teoría del Conocimiento (Gay Bochaca, 2001: 33).

- Grupo 11: Género, involucra asignaturas enfocadas específicamente en la relación entre el derecho y el género, como el Derecho de Familia con enfoque de género o el género en sí mismo (Nieves, 2011: 10). 
- Grupo 12: Humanidades, Ciencias Sociales y del Comportamiento, incluye asignaturas como Antropología, Sociología, Ciencia Política, Economía, Historia, Psicología, entre otras, contempladas en el campo específico 022 como en el o31 de la CINE (Unesco, 2017).

- Grupo 13: Investigación, tiene que ver con las asignaturas enfocadas en el desarrollo de habilidades y competencias investigativas, como métodos de investigación científica, jurídica o social.

- Grupo 14: Matemáticas y Estadística, hace referencia a asignaturas con esas denominaciones correspondientes al campo específico 054 de la CINE (Unesco, 2017).

- Grupo 15: Pluralismo e Interculturalidad abarca asignaturas que destaquen el carácter plural del derecho en el Ecuador, o los aspectos de interculturalidad en aplicación de la justicia.

- Grupo 16: Práctica abarca asignaturas enfocadas en la práctica del derecho que en ocasiones se presentan como clínicas o prácticas jurídicas en materias específicas, como Derecho Civil, Penal, Laboral, Constitucional (García Arellano, 2004: 21).

- Grupo 17: Ramas Específicas del Derecho y Legislación se refiere tanto a campos muy especializados del Derecho como la rama energética o informática, así como a asignaturas cuya denominación se refiere a los cuerpos normativos, como la Ley de Aduanas o la Ley de Control del Poder del Mercado.

- Grupo 18: Técnicas Auxiliares se refiere a las asignaturas que buscan desarrollar destrezas prácticas a través de técnicas específicas, como el manejo de herramientas informáticas, la oralidad jurídica o la oratoria forense.

Entre los hallazgos del análisis se puede observar que en el grupo 1, Educación Comercial y Administración, hay trece universidades que cuentan con asignaturas relacionadas. Cabe señalar que únicamente una de estas asignaturas tiene que ver con el sector público y es ofrecida por una universidad pública; las demás se concentran en la actividad administrativa privada y en su mayoría forman parte del plan de estudios de universidades privadas.

El grupo 2, Artes, tiene la más baja presencia en el currículum de las carreras de Derecho: apenas una universidad cuenta con una asignatura en ese campo, lo cual muestra la poca relevancia que tiene para las instituciones el diálogo con esta disciplina en la formación jurídica.

El grupo 3, relacionado con la ciencia jurídica, tiene asignaturas brindadas por 24 instituciones de educación superior. Las asignaturas que más se repiten son Introducción al Derecho y Teoría del Derecho con 17 y 9 universidades, respectivamente. 


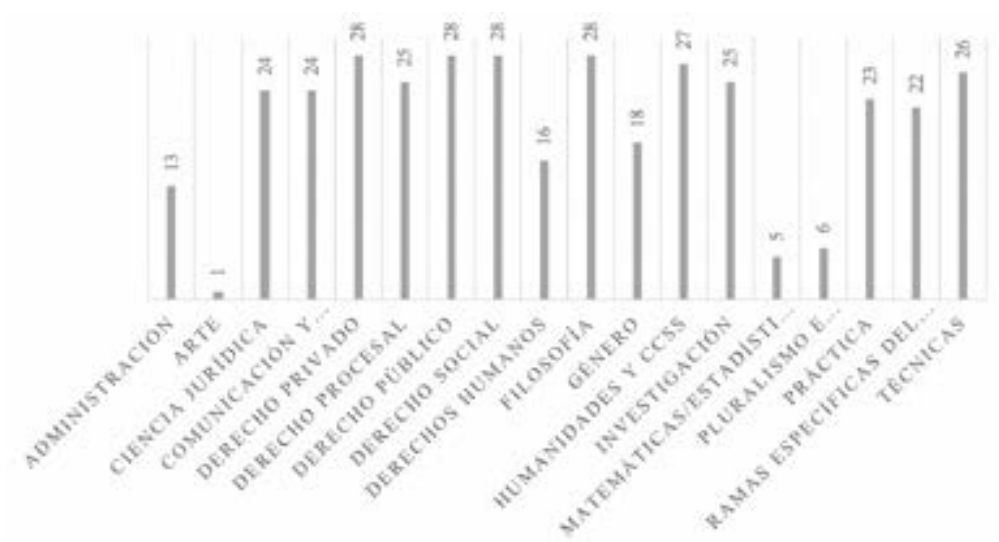

Gráfico 1. Número de universidades por grupo de asignaturas. Elaboración propia en base a los sitios web de las instituciones educativas.

Aquellas que tienen menos presencia son Ciencia Jurídica y Teoría de la Norma. En este grupo se encuentran asignaturas que guardan relación con el componente de Introducción al Derecho y Filosofía del Derecho que el CEAACES evalúa en su examen de evaluación de carreras. Sin embargo, cuatro instituciones no formarían a los estudiantes en este componente.

El grupo 4, Comunicación y Lenguaje, cuenta con materias que son compartidas por 24 universidades, con lo cual se puede decir que las universidades dan importancia a las capacidades comunicativas que pueda adquirir el estudiante para el ejercicio de su profesión, tanto oral como escrita y en otras lenguas. Destaca una única asignatura impartida en idioma inglés, Writing and rethoric, brindada por una universidad privada de la Sierra.

Sobre el grupo 5, Derecho Privado, se observa que todas las instituciones tienen asignaturas de este conjunto. La principal es Derecho Civil que comparten todas las instituciones. Más de 24 universidades tienen 4 niveles en los que se imparte Derecho Civil, y 2 tienen hasta 8 niveles. Podría afirmarse que, de todas las materias del currículum de la carrera de Derecho, esta es la más importante y tradicional. A esta asignatura le sigue la de Derecho Mercantil con 24 instituciones que la imparten. Esto permite concluir que la formación jurídica en el Ecuador da mayor importancia al Derecho Privado, a las relaciones jurídicas vinculadas a la propiedad y al comercio. Otra asignatura con un buen número de instituciones que la enseñan es la de Métodos Alternativos de Resolución de Conflictos, con 18 instituciones. Si bien esta asignatura propone buscar una cultura de paz en la que el sistema no busca la justicia como fin, sino la conclusión de conflictos que permite la descongestión del sistema público de justicia (Gonzalo y otros, 2011: 12), también muestra rasgos privatizadores de la justicia, que incluye barreras económicas para su acceso y enfocada en los conflictos de las corporaciones (Díaz, 2004: 651). 
En cuanto al grupo 6 sobre Derecho Procesal, 25 universidades cuentan con asignaturas vinculadas a este grupo, concentradas en el campo civil, penal, constitucional $y$, en algunos casos, laboral, mercantil y societario.

El grupo 7, Derecho, Público se imparte en todas las instituciones analizadas. La asignatura que coincide en todas las universidades es Derecho Constitucional. Esto se explica por el predominio del modelo de Estado constitucional impulsado desde la segunda mitad del siglo XX en la tradición del civil law. Al menos 10 universidades tienen dos niveles en las que se imparte Derecho Constitucional, y en dos de ellas tiene tres niveles. A esta asignatura le siguen las de Derecho Administrativo, Penal, y Tributario con 27 instituciones. Cabe señalar que las universidades que no cuentan con materias con esta denominación sí imparten formación relacionada a través de otras denominaciones, como Teoría del Estado o Ciencias Penales, por lo que podría afirmarse que las asignaturas mencionadas constituyen el núcleo de la formación del Derecho Público. En el caso del Derecho Penal, 24 instituciones tienen 2 niveles dedicados a su estudio y 6 tienen 3 niveles, con lo cual se puede concluir que, de este grupo, es la asignatura a la que las universidades le conceden mayor importancia. Las asignaturas que menor presencia tienen entre las instituciones son Derecho Penitenciario, que podría estar inmerso en los contenidos de las asignaturas de Derecho Penal; Derecho Municipal que, de la misma forma, podría estar dentro de las asignaturas de Derecho Administrativo; Derecho Internacional Económico, que podría formar parte del Derecho Internacional; y Derecho Aduanero, que podría formar parte del Derecho Tributario u otra asignatura sobre Derecho Comercial o Económico.

Un aspecto que vale destacar es la poca importancia que se concede al Derecho de Integración. Considerando que el Ecuador es parte de algunos procesos de integración, como la Comunidad Andina de Naciones, Unasur y CELAC, apenas ocho instituciones cuentan con una asignatura específica sobre esto, aunque es posible que su contenido esté inserto en la asignatura de Derecho Internacional Público.

Otra asignatura con la que pocas universidades cuentan es aquella relacionada con el ejercicio de la judicatura: solo seis instituciones forman a los juristas en este campo. Sin embargo, la literatura señala que es una necesidad del perfil profesional contar con las destrezas, habilidades y conocimientos que requieren los funcionarios judiciales, específicamente los jueces y fiscales (Pásara, 2009: 104). Pese a ello, de los resultados del análisis se observa que a nivel del plan de estudios esta no es una prioridad en las carreras de Derecho. Un argumento en defensa a este aparente descuido es que los conocimientos están distribuidos en otras asignaturas, como las de Derecho Procesal, Interpretación o Argumentación. Alcanzar conclusiones sobre esto rebasa el objeto de este estudio, pues sería necesario indagar en los contenidos de esas asignaturas para identificar qué orientación tienen.

Por otro lado, es interesante como cinco universidades de la región Costa, ubicadas en ciudades con puertos, han incluido en sus planes de estudios la asignatura de 
Derecho del Mar o Derecho Marítimo. Esto puede responder a la importancia del rol de su actividad en la economía local, que abre un espacio de ejercicio de la profesión.

El grupo 8, sobre el Derecho Social, también está presente en todas las instituciones. La principal asignatura de este grupo es el Derecho Laboral, seguido del Derecho Ambiental. Al igual que en el caso del Derecho Marítimo analizado antes, el Derecho Agrario que se ha ubicado en este grupo se observa en el plan de estudios de universidades ubicadas en provincias con una importante economía agrícola, tanto de la Sierra como de la Costa.

El grupo 9, Derechos Humanos, cuenta con asignaturas que comparten 16 de las 28 universidades; sin embargo, es posible que, en las demás instituciones, aspectos sustanciales de los derechos humanos se encuentren subsumidos en las asignaturas relacionadas con el Derecho Constitucional.

En el grupo 10, Filosofía, existen asignaturas compartidas por todas las universidades que cuentan con la carrera de Derecho. La asignatura que más se repite es Filosofía del Derecho, con 23 universidades que la ofertan. Esto nuevamente guarda relación con los componentes del examen de evaluación de carreras del CEAACES. Las asignaturas que menor repetición tienen son Teoría del Conocimiento, Epistemología de las Ciencias y Bioética.

El grupo 11, denominado Género, incluye asignaturas que involucran tanto el Derecho de Familia y Género como los estudios de género en sí mismos. Se observa que 18 instituciones tienen asignaturas relacionadas con el Derecho de Familia y Género y solamente dos cuentan con estudios de género.

En el grupo 12, Humanidades y Ciencias Sociales, la asignatura que mayor número de universidades comparte es la de Historia del Derecho o Derecho Romano, con 22 instituciones, seguida por asignaturas sobre el contexto socioeconómico, cultural y ambiental, con 13 instituciones, y Sociología del Derecho, con 11 instituciones. Hay 14 asignaturas de este grupo que tienen una presencia menor en las instituciones; entre estas se encuentran Mundología, Realidad y Conocimiento, Psicología, Humanismo Integral, Cosmos, y la mayoría de las instituciones que las consideran son de naturaleza jurídica privada. Estas cifras evidencian un consenso de las universidades sobre las asignaturas nucleares de las humanidades y las ciencias sociales que debiera tener el plan de estudios de la carrera de Derecho, así como también se puede observar que las asignaturas menos repetidas muestran quizás una exploración menos tradicional por parte de las universidades privadas, que buscan una formación en aspectos epistemológicos y de autoconocimiento.

El grupo 13, Investigación, se brinda en 25 instituciones. Se espera que al ser la investigación una de las funciones principales de la universidad y una competencia indispensable para el aprendizaje y la formación a lo largo de la vida, así como la solución de problemas en el campo profesional, alguna asignatura relacionada a enseñar a investigar se imparta en toda carrera de Derecho. Contrario a esto, se verifica que 
tres instituciones no lo hacen; cinco instituciones cuentan con asignaturas de metodología de la investigación científica y, a la vez, con otra que por su denominación se intuye que profundizan en el método profesional del campo jurídico.

En cuanto al grupo 14, Matemáticas y Estadística, se observa que apenas cinco universidades brindan asignaturas en estos campos del conocimiento, lo cual da cuenta de la poca importancia que se da al diálogo interdisciplinario. Nuevamente se observa que aquellas instituciones que ofertan estas asignaturas son privadas, lo que da pistas de que la formación en las instituciones públicas es más tradicional y disciplinaria.

El grupo 15, sobre Pluralismo e Interculturalidad, tiene muy baja participación, lo cual indica que las universidades no han interiorizado el carácter plural del derecho instaurado en la Constitución del año 2008, y que ya tenía alguna presencia en la Constitución de 1998. Solamente seis universidades cuentan con asignaturas relacionadas con este grupo que, podría pensarse, están ubicadas en las áreas geográficas con mayor presencia de pueblos y nacionalidades indígenas, afroecuatorianos o montubios, pero no es así, pues la mitad están la ciudad de Quito.

El grupo 16, denominado Práctica, tiene asignaturas que comparten 23 universidades. Estas asignaturas se concentran principalmente en el práctica civil y penal, seguidas por la laboral y constitucional. Muchas de estas asignaturas tienen una denominación de clínicas, que responde al impulso global para el aprendizaje del derecho a través de la educación con casos. Aunque el examen del CEAACES no considera aspectos de carácter práctico, la cantidad de universidades que oferta asignaturas de este grupo muestra que hay un acuerdo respecto a que el desarrollo de actividades prácticas es necesario para la formación de los abogados y abogadas.

El grupo 17, Ramas Específicas del Derecho y Legislación, con 22 universidades que tienen asignaturas relacionadas, muestra que una buena parte de instituciones continúa enseñando leyes y no Derecho. Once universidades enseñan directamente legislación, como leyes de tránsito, aduanas, Código de Comercio, Código Civil, Código Penal, Ley de Garantías Jurisdiccionales. Este fenómeno se presenta en las universidades de provincia y no en las ubicadas en Quito, Guayaquil. Lo cual indica que la enseñanza de corte positivista y formalista es más profunda en las zonas periféricas.

El grupo 18, Técnicas Auxiliares, está presente en 26 universidades, e incluye herramientas informáticas, criminalística, tramitología. Al respecto, varias de estas asignaturas son indispensables para el desarrollo de habilidades y destrezas útiles en el campo profesional, como por ejemplo las herramientas informáticas. No obstante, llama la atención la mera existencia de la asignatura denominada Tramitología: esto da señales que las instituciones que mantienen esta materia miran al perfil del profesional jurídico como un facilitador de trámites. 


\section{Conclusiones}

\section{Educación jurídica tradicional}

Tal como se puede ver del análisis de los diferentes grupos de asignaturas, la carrera de Derecho en el Ecuador se concentra en enseñar Derecho Civil, Penal, Constitucional, Administrativo, Laboral y Tributario, pues son estas las asignaturas que constituyen el común denominador en todas las instituciones de educación superior que ofertan Derecho. El Derecho Procesal, Mercantil, la Filosofía del Derecho, Historia del Derecho o Derecho Romano, el Derecho Internacional y la investigación ocupan un segundo lugar, y en tercer lugar estás las asignaturas de técnicas auxiliares. Son escasas las asignaturas que desarrollan una comprensión pospositivista y crítica del derecho, como la argumentación jurídica, la interpretación, el pensamiento crítico jurídico. Si bien puede ser que estos enfoques sean abordados en las asignaturas de Filosofía o Teoría del Derecho, o a través de una mirada transversal, hay algunas instituciones que dan mayor importancia a estos temas al crear asignaturas independientes que permitan tener el tiempo suficiente para su profundización. Por ello puede concluirse que la educación jurídica en el Ecuador es predominantemente de corte tradicional en los modelos dos y cuatro de la clasificación de Gordon (2002), esto es, se concentra en la enseñanza de las leyes y de la ciencia jurídica, lo cual concuerda con lo que sucede en la región (Pérez Perdomo, 2007: 260).

Las asignaturas centrales de las carreras de Derecho del Ecuador coinciden con los contenidos del examen de evaluación del CEAACES. Por el alcance del estudio no se puede identificar si las universidades han adaptado los planes de estudio a dicho examen o si el examen fue diseñado sobre las asignaturas que ya existían en los planes de estudio. No obstante, el delineamiento de los contenidos de la evaluación pudo ser la oportunidad para impulsar una reflexión sobre el tipo de profesionales jurídicos que requiere el país, corriendo el riesgo de que los contenidos del examen limiten la posibilidad de realizar propuestas de educación jurídica innovadoras o diferenciadoras. Sucede entonces aquello que Pérez Perdomo señala como un fenómeno en la región: «Si la educación tradicional es todavía mayoritaria en el sistema, es probable que los equipos evaluadores asocien la buena educación jurídica con una educación tradicional» (2007: 260).

\section{Escaso diálogo con otras disciplinas}

Las asignaturas que son escasas se concentran en las humanidades y ciencias sociales que permiten conocer al derecho dentro del contexto en el que se desarrolla y aplica, así como tener una lectura reflexiva y crítica del mismo, como Sociología, Ética profesional, Política, Antropología y Economía. Solo once universidades cuentan con estas asignaturas, es decir, un poco más de la tercera parte. 
Si el diálogo con este campo del conocimiento es pobre, lo es aún más con campos que se podrían considerar como lejanos, pero que son indispensables para contar con una formación integral, como las matemáticas, la estadística y el arte.

Pérez Perdomo (2007: 55), luego de más de cuarenta años de dedicarse a la educación jurídica alcanza la conclusión de que los mejores profesionales jurídicos son los que pueden trabajar de manera colaborativa e interdisciplinaria, con capacidades investigativas y comunicativas, pues tienen más posibilidades de adaptarse al actual mundo cambiante. Además, afirma que la educación tradicional no forma este tipo de abogado.

De la información analizada se puede concluir que la educación jurídica en el Ecuador en su conjunto forma profesionales del derecho espectadores, con poco espacio para la reflexión crítica. Los conocimientos, destrezas y actitudes que se ofertan en las carreras para responder al actual paradigma constitucional son pocos.

Poca importancia a los enfoques de derechos humanos, género e interculturalidad

Los enfoques poco incorporados son los de género e interculturalidad. Sobre esto podría sostenerse que están transversalizados. Tal hipótesis no puede comprobarse a través de este estudio porque su alcance se limita al análisis de las denominaciones de las asignaturas, pero no a sus contenidos. Sin embargo, varios autores sostienen que es necesario que estos temas se aborden tanto transversal como específicamente por la transcendencia que tienen en la actualidad, sobre todo en un Estado de derecho plurinacional con reconocimiento de la justicia indígena (Nieves, 2011: 19-20; SENESCYT, 2015: 91; Aba, 2014: 188).

\section{Visión predominantemente liberal de la educación jurídica}

Tal como se ha señalado, la asignatura a la que mayor tiempo se le dedica durante la carrera es al Derecho Civil, núcleo del Derecho Privado. Esto evidencia que la mayor influencia de la formación jurídica en el Ecuador es la de la tradición liberal del siglo XIX, y no la del paradigma del Estado de derecho y justicia del constitucionalismo del Ecuador. Además, al menos la mitad de las instituciones de educación superior incorporan asignaturas relacionadas a la administración y el emprendimiento, con lo cual se puede concluir que se espera que el graduado esté preparado para el ejercicio liberal de la profesión. Esto además tiene relación con el predominio de un perfil litigante del abogado o abogada, dejando de lado la importancia de otros roles como el de juez, fiscal, académico o asesor. 


\section{Referencias}

АвA, Ana (2014). «Implicaciones de la Ley de Igualdad en las Universidades. Actuaciones Específicas de la UDC: El Plan de Igualdad». En Ana López (editora), II Xornada Universitaria En Xénero. Santiago de Compostela: Universidad de la Coruña; Universidad de Vigo; Universidad de Santiago de Compostela.

Aguirre, Manuel Agustín (1973). La Segunda Reforma Universitaria. Quito: Universidad Central del Ecuador.

Betancourt, Laura y Enrique Prieto (2017). «Educación del derecho internacional en Bogotá: un primer diagnóstico a partir de los programas de clase y su relación con las epistemologías de no conocimiento». Revista Derecho del Estado, (39): 53-89.

BLoom, Benjamin et al. (1956). Taxonomy of Educational Objectives: The Classification of Educational Goals. New York: David McKay Company.

CEAACES, Consejo de Evaluación, Acreditación y Aseguramiento de la Calidad de la Educación Superior (2017). Guía metodológica de orientación al estudiante. Quito: CEAACES.

DíAz, Miguel (2004). Arbitraje, Privatización de La Justicia. Editorial Themis.

Díaz Ocampo, Eduardo (2018). «La formación del profesional en la Educación Superior en la carrera de Derecho en Ecuador». Revista Espacios. 39 (29): 1-11.

De Mello Massimino, Daniel y Danielle Anne Pamplona (2015). ««Saberes» de Morin na Educação Jurídica: Caminhos à Educação para o desenvolvimento sustentável». Opción, 31 (3): 446-469.

Donnelly, Jack (2013). Universal Human Rights in Theory and Practice. Third. New York: Cornell University Press.

Elgueta Rosas, María Francisca y Eric Eduardo Palma González (2014). «Una propuesta de clasificación de la clase magistral impartida en la Facultad de Derecho». Revista Chilena de Derecho, 41 (3): 907-924.

Estarellas Velázquez, Carlos Ernesto y René Manuel Velázquez Ávila (2018). «Las relaciones internacionales de frente a la globalización de la educación superior ecuatoriana» Revista Redes, 19 (10): 16-25.

García Arellano, Carlos (2004). «Las grandes divisiones del derecho». Revista de la Facultad de Derecho de México, 54, 242: 11-32.

Gay Bochaca, José (2001). Curso de filosofía. Madrid: Ediciones Rialp.

GinÉ, Nuria (2009). «Cómo mejorar la docencia universitaria: El punto de vista del estudiantado». Revista Complutense de Educación, 20 (1): 117-134.

Gordon, Robert (2002). Distintos modelos de educación jurídica y las condiciones sociales en las que se apoyan. SELA (Seminario en Latinoamérica de Teoría Constitucional y Política) Papers. Paper 6. Disponible en http://digitalcommons.law. yale.edu/yls_sela/6 
GonZÁLEZ Galván, Jorge Alberto (2013). «Legal Education, Research and Intelligents Human Rights». Boletín Mexicano de Derecho Comprado, 137: 499-527.

GonzÁlez IbáÑEZ, Joaquín (2012). «Pedagogía jurídica, Estado de derecho y derechos humanos». Anuario Colombiano de Derecho Internacional, 5: 1-28.

Herrero, Julio César y María del Mar Perelló Roselló (2017). «La enseñanza del protocolo y la organización de eventos en el espacio europeo de educación superior: análisis comparado de los planes de estudio». Estudios sobre el Mensaje Periodístico, 23 (1): 437-455.

Herrera, Sindy, Germán González y Yarina Paniagua (2018). «Aspectos pedagógicos y curriculares por considerar en el rediseño de un plan de estudios de posgrado con énfasis en docencia universitaria». Revista Electrónica Educare, 22 (2): 1-19.

INEC, Instituto Nacional de Estadística y Censo (2017). «Tras las cifras de Quito». Disponible en http://www.ecuadorencifras.gob.ec/tras-las-cifras-de-quito/.

KeNNEDY, Duncan (2004). «La educación legal como preparación para la jerarquía». Academia, 3: 117-147.

Lazo González, Patricio (2011). «Formación jurídica, competencias y métodos de enseñanza: premisas». Ius et Praxis, 17 (1): 249-262.

Lово, Mercedes (2017). «Relación de la teoría y la práctica en la enseñanza del derecho». Revista Espacios, 38 (45): 1-5.

Londoño Toro, Beatriz (2016). «Los cambios que requieren las clínicas jurídicas iberoamericanas. Estudio de caso en seis países de la Rrgión». Boletín Mexicano de Derecho Comparado, 49, 146: 119-148. https://doi.org/10.22201/ IIJ.24484873E.2016.146.10508

Mourão, Lucía, Rita Martins, Carla Vieira y Solange L’Abbate (2007). «Análise institucional e educação: Reforma curricular nas universidades pública e privada». Educação \& Sociedade, 28 (98): 181-210.

Nieves, María (2011). «Los estudios de género en los grados en derecho». Revista de Educación y Derecho, 3: 1-22.

NúÑEZ VAQUero, Álvaro (2009). "Ciencia Jurídica». En Jorge Luis Fabra Zamora (editor), Enciclopedia de Filosofía Y Teoría Del Derecho. México: Universidad Nacional Autónoma de México.

Osorio, Aura Ximena (2013). «Hacia la excelencia en la educación jurídica». Universitas, 126: 11-14.

PÁsara, Luis (2009). «Desafíos de La Transformación de La Justicia En América Latina». En Santiago Ávila y Luis Andrade (editores), La Transformación de La Justicia. Quito: Ministerio de Justicia, Derechos Humanos y Cultos.

Pérez Lledó, Juan Antonio (2001). La enseñanza del derecho en Estados Unidos. Alicante: Biblioteca Virtual Miguel de Cervantes. 
Pérez Perdomo, Rogelio (2007). «Educación jurídica, abogados y globalización en América Latina». Abogacía y Educación Legal, 9: 255-275.

Pinilla Rodríguez, Diego, Héctor Reinoso Vásquez y María Eugenia López MeriNo (2017). «Complejidad, formación y transformación social. Algunas anotaciones». Revista Jurídicas, 14(1): 87-101.

Solari, Enzo (2015). «La filosofía jurídica y su enseñanza». Revista Chilena de Derecho, 42 (1): 369-39o.

SuÁrez, Eloy Emiliano (2004). Introducción al derecho. Buenos Aires: Universidad Nacional del Litoral.

VerEs, Ernesto (2002). «Los nuevos planes de estudio y su incidencia sobre la edad de finalización de los estudios superiores. El caso de la Universidad de Valencia». Relieve. Revista Electrónica de Investigación y Evaluación Educativa, 8 (1): 30-48.

WITKER, Jorge (2007). «La enseñanza clínica como recurso de aprendizaje jurídico». Academia, 10: 181-207.

UnEsCo (2013). Revisión de la Clasificación Internacional Normalizada de la Educación: Campos de la Educación y la Formación. París: Unesco.

\section{Sobre los autores}

Rina Pazos Padilla es abogada y magíster en Derecho Administrativo, candidata a $\mathrm{PhD}$ en Ciencias Jurídicas Generales por la Universidade do Minho de Portugal. Actualmente está afiliada a la Universidad Tecnológica Equinoccial del Ecuador. Su correo electrónico es rina.pazos@ute.edu.ec.

Jorge Fabara Espín es abogado y candidato a $\mathrm{PhD}$ en Modernidades Comparadas por la Universidade do Minho de Portugal. Su correo electrónico es id7168@ alunos.uminho.pt. 
La Revista Pedagogía Universitaria y Didáctica del Derecho (RPUDD) es una publicación científica semestral que contribuye a la reflexión multidisciplinaria sobre pedagogía universitaria y didáctica del derecho, para la formación y consolidación de esta área de investigación; así como a la difusión de prácticas innovadoras en la enseñanza-aprendizaje del derecho considerando el contexto nacional e internacional. Es una publicación electrónica internacional con una codirección entre Brasil y Chile.

\author{
DIRECTORA \\ María Francisca Elgueta Rozas \\ Universidad de Chile \\ DIRECTOR \\ Renato Duro Dias \\ Universidad Federal de Rio Grande, Brasil \\ SITIO WEB \\ pedagogiaderecho.uchile.cl \\ CORREO ELECTRÓNICO \\ rpedagogia@derecho.uchile.cl \\ LICENCIA DE ESTE ARTÍCULO
}

Creative Commons Atribución Compartir Igual 4.o Internacional

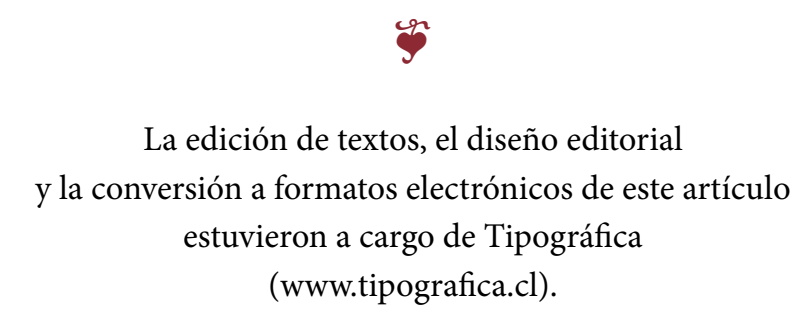

